\title{
Continuous Production of Silver Nanoparticles and Process Control
}

\author{
Olga Długosz ${ }^{1}$ (D) Marcin Banach ${ }^{1}$
}

Received: 13 November 2018/ Published online: 13 February 2019

(C) The Author(s) 2019

\begin{abstract}
The purpose of this research was to prepare silver nanoparticles in a tubular reactor using tannic acid as both a reducing agent and a stabiliser. The stability of the process was determined using statistical analysis with control charts. The qualitative analysis of the resulting colloidal silver nanoparticles was investigated using UV-Vis spectroscopy, dynamic light scattering method and the transmission electron microscopy. Particles with average sizes ranging from 14 to $60 \mathrm{~nm}$ were obtained. Stability of the process was achieved mainly through the use of 0.5 Tannic acid/ $\mathrm{AgNO}_{3}$ molar ratios and temperature ranging from 20 to $45^{\circ} \mathrm{C}$. The proposed technique is consistent with the principles of green chemistry and can be an alternative for traditional nanosilver synthesis.
\end{abstract}

Keywords Tubular reactor $\cdot$ Silver nanoparticles $\cdot$ Control charts $\cdot$ Green chemistry

\section{Introduction}

Due to the growing interest in products composed of components at the nano scale, the demand for the production of nanoparticles, including mainly nanosilver, is increasing [1]. Currently, due to the relatively small quantities, nanosilver is produced in batch processes using mostly reduction methods. These methods do not require harsh process conditions, such as high temperature, and the process is relatively fast [2]. The factors affecting the quality of the process include: the type of nanosilver precursor used, the reducer and stabiliser, and physicochemical parameters such as temperature and $\mathrm{pH}$ of the solution [3].

In the past decade, environmentally benign synthesis of nanoparticles has received increasing attention. According to the principles of green chemistry, processes are being developed and implemented to reduce or eliminate the use and production of hazardous substances. In the production of silver nanoparticles, sodium borohydride [4], hydrazine [5], or formaldehyde [6] are mostly used as reducing

Olga Długosz

odlugosz@chemia.pk.edu.pl

1 Faculty of Chemical Engineering and Technology, Cracow University of Technology, Warszawska St. 24,

31-155 Cracow, Poland agents. However, obtaining nanosilver using the aforementioned compounds has a negative impact on the environment. These substances are highly toxic and some are also carcinogenic. Instead, substances of natural origins that are not toxic to the environment are increasingly used as a reducer or stabiliser. For instance, gallic acid [7], sodium citrate, and glucose are environmentally friendly substances. Some of them act both as reducing and stabilising agents, which decreases the number of compounds used in the synthesis process. An example of such substance is tannic acid belonging to tannins, derivatives of polyphenols. Combination of reducing and stabilising properties results from its construction. Tannic acid consists of a glucose molecule, to which polygalloyl ester chains are attached. Under basic conditions tannic acid hydrolyses into glucose and gallic acid molecules. Glucose is widely known as a stabiliser agent whereas it is a weaker reducer, compared to widely known reducers, sodium borohydride, among others. In contrast, gallic acid has good reducing properties but low stabilising properties. In this way, both compounds act synergistically ensuring good conditions for obtaining nanoparticles [8, 9]. Tannic acid takes part in redox reactions by forming quinones and donating electrons. The presence of electrons allows to reduce metal ions in metal salts to form corresponding metal nanoparticles. The reaction mechanism of tannic acid-based reduction of silver ions is outlined in Fig. 1. The activation of the acid takes place in the presence of 
Fig. 1 Reaction mechanism of tannic acid-based reduction of silver salts [9]
Phenolic form

Quinone form

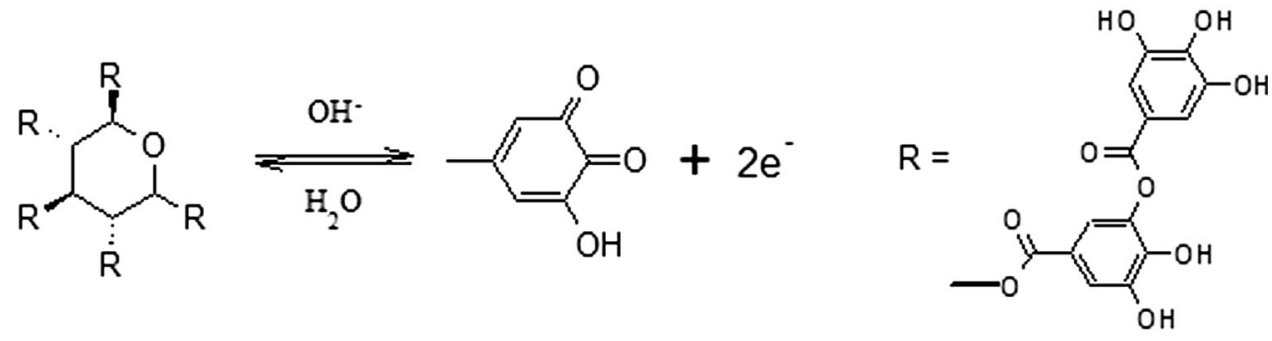

\section{$\mathrm{Ag}^{+}+\mathrm{e}^{-} \longrightarrow \mathrm{Ag}^{0}$}

hydroxyl groups, hence the solution of the hydroxide, e.g. $\mathrm{NaOH}$, is added to the system [9].

Currently, most methods of nanosilver production are carried out batch-wise in the laboratory. The disadvantages of that solution are poor reproducibility and long process time. An alternative to a batch process is to feed the reactants continuously into the reactor. A limited number of nanoparticles are being created in a continuous process. However, continuous systems are becoming more and more popular, as is apparent from the amount of articles related to this topic. Chou et al. carried out studies on the continuous precipitation of silver nanoparticles [10]. They investigated the effects of the concentrations of the silver precursor and stabiliser and the effect of the flow rates of the reacting solutions. The synthesis reaction was carried out in a simple microchannel reactor. Baber et al., using microfluidical coaxial flow reactor, investigated effect of flow rate and concentrations of reagents on size and distribution of silver nanoparticles. They achieved monodispersed nanoparticles with a spherical shape and particle size less than $10 \mathrm{~nm}$ [11]. Tai et al. [12] used a spinning disk reactor with glucose used as a reducing agent. They achieved particles in range from 4 to $10 \mathrm{~nm}$, but the conversion was only $70 \%$. In other studies, different constructions of the reactors were investigated, for example a narrow channel reactor [13], a T-shaped mixer with a tubular reactor [14], and a microchannel reactor [10]. He and Liu synthesised nanosilver in an organic solvent using silver acetate as the precursor with a capillary microflow reactor in a high-temperature oil bath at $170{ }^{\circ} \mathrm{C}$ [15]. Deshpande and Kulkarni analysed kinetics of silver nanoparticles synthesis using citrate reduction. They used several reactor configurations among others segmented flow reactors and 3D flow reactor. The researches established particle size distribution and conversion rate for nanosilver production process [16]. Interesting results were presented by Cristaldi et al. who, using the method combining 3D printing of master moulds with the sealing of the PDMS channels' replica, made a microreactor for the production of organic and inorganic nanoparticles. To demonstrate the usability of the technique, reactors were applied to the production of silver nanoparticles and liposomes. The advantage of the work was the development of a cost-effective and easy-to-perform fabrication method for continuous flow reactors production [17].

The aim of this work was to study the production process of nanosilver in a tubular reactor using tannic acid as both a reducing agent and a stabiliser. In the research, the effect of the tannic acid concentration and the effect of temperature on the final properties of silver nanoparticles were investigated. Reactor stability was also checked using control charts.

\section{Materials and Methods}

\section{Materials}

Silver nitrate (99.9\%, Sigma-Aldrich) and tannic acid (Sigma-Aldrich) were used in this work to synthesise nanosilver colloids. The concentration of the precursor solution was $500\left[\mathrm{mg} \mathrm{Ag}^{+} / \mathrm{dm}^{3}\right]$. The molar ratio of tannic acid to $\mathrm{Ag}^{+}$(nT/nAg) was examined in range from 0.2 to 0.8 . The initial $\mathrm{pH}$ of the solution was 10 and it was adjusted by adding $0.20-0.25 \mathrm{M} \mathrm{NaOH}(98.8 \% \mathrm{POCH})$.

\section{The Continuous Production of Silver Nanoparticles}

The continuous production process of silver nanoparticles was carried out in a glass tubular reactor. A schematic diagram of the experimental setup is presented in Fig. 2. Parameters of the reactor and flow rate of each reagent are reported in Table 1. Solutions: silver nitrate, tannic acid and sodium hydroxide were fed with piston pumps (Micropump HPLH, Conbest). Flow rates of the reagents were constant and the reagents volume ratios were equalled 9:1:1 for $\mathrm{AgNO}_{3}$, tannic acid and $\mathrm{NaOH}$ solutions, respectively (values of flow rates of reagent are compared in Tab. 1). Due to the laminar flow of mixture inside the 


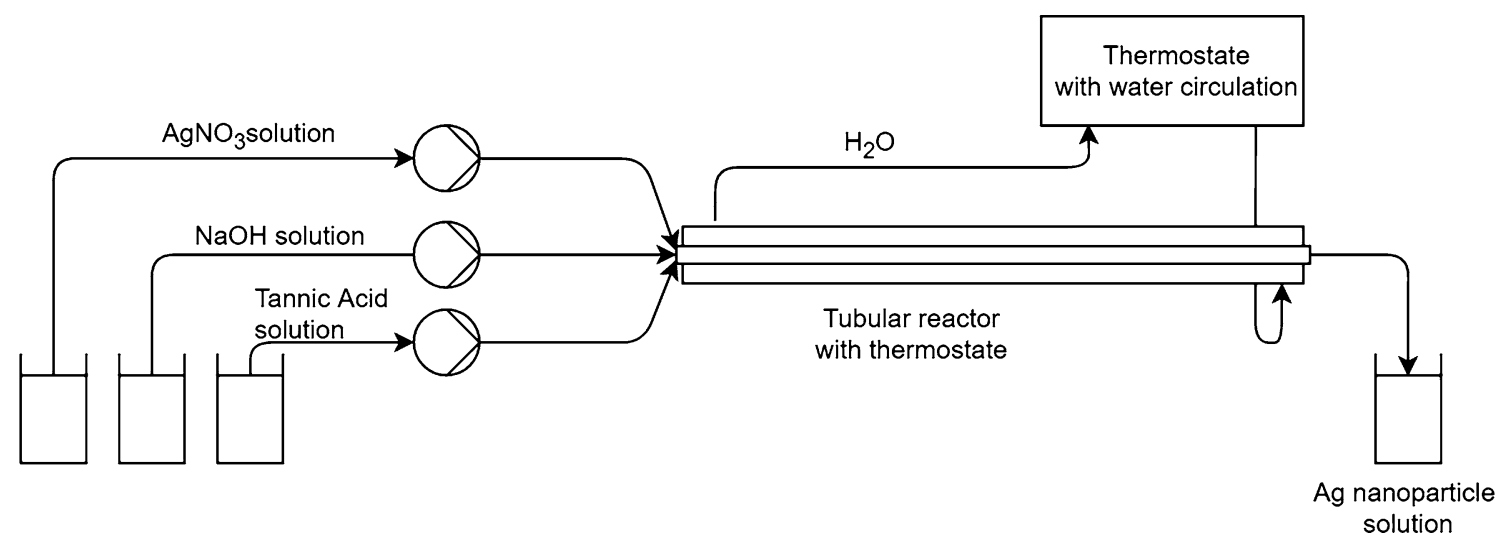

Fig. 2 Schematic diagram of the experimental setup for the synthesis of silver nanoparticles in tubular reactor

Table 1 Parameters of the tubular reactor used for production of silver nanoparticles

\begin{tabular}{ll}
\hline Parameter & Value \\
\hline Length of the reactor $L_{r}[\mathrm{~m}]$ & 0.40 \\
diameter of the reactor $d[\mathrm{~m}]$ & $2.5 \cdot 10^{-2}$ \\
Flow rate of silver nitrate solution $F v_{\mathrm{AgNO}_{3}}[\mu \mathrm{l} / \mathrm{s}]$ & 270 \\
Flow rate of tannic acid solution $F v_{\text {tannic }}[\mu \mathrm{l} / \mathrm{s}]$ & 30 \\
Flow rate of sodium hydroxide solution $F v_{\mathrm{NaOH}}[\mu \mathrm{l} / \mathrm{s}]$ & 30 \\
Operative total flow rate $F v[\mu \mathrm{l} / \mathrm{s}]$ & 330 \\
\hline
\end{tabular}

reactor $(16.44<\operatorname{Re}<16.78)$, the inlets of the individual reactants were directed at $60^{\circ}$. The construction allowed to mix reagents in high degree, without adding additional improvements. The reactor consisted of an internal glass tube in which the synthesis reaction took place and an external glass tube in which water circulated, maintaining constant temperature conditions. Water has heated by an external thermostat with water circulation.

For the analysis of processes, each measurement of the synthesis of nanoparticles was tested for $90 \mathrm{~min} .10 \mathrm{ml}$ of the sample with the nAg suspension was collected every $5 \mathrm{~min}$ and analysed by UV-Vis Spectroscopy. In this study, the effect of the molar ratio of tannic acid to $\mathrm{AgNO}_{3}$ and the effect of temperature (in the range of $20-70{ }^{\circ} \mathrm{C}$ ) for the creation of nanosilver was determined.

The characteristics of the nanoparticles were examined by UV-Vis spectroscopy. The analysis of UV-Vis spectroscopy was performed using Rayleigh UV18000 spectrophotometer at $1 \mathrm{~nm}$ resolution. Concentration of silver ions in the final solution was determined by the potentiometric method using a silver-sulphate and a reference electrode (Hydromet company) connected to the multimeter CX-701 by Elmetron. The degree of conversion of silver ions was determined as the difference between the initial concentration and the final concentration of silver ions. Conversion of silver ions was evaluated by the following formula:

$\alpha_{A g^{+}}=\frac{C_{0 A g^{+}}-C_{A g^{+}}}{C_{0 A g^{+}}} \cdot 100 \%$

The resulting monodisperse nanoparticles of silver colloids were examined using electrophoretic dynamic light scattering (DLS) (Malvern Instruments, ZS-90 and Brookhaven, zetaPALS). To illustrate the size and shape of particles, microphotographs of obtained samples were taken using transmission electron microscopy technique (TEM) with EDX and elemental mapping mode using Tecnai Transmission Electron Microscope, F20 X-Twin, FEI Europe.

\section{The Control Charts}

The stability of the reactor was tested with two types of control charts. The control charts on $\bar{X}$ and the moving range $\mathrm{R}$ were created by analysing the average diameter of nanoparticles determined by the DLS method. The multidimensional Hotelling $\mathrm{T}^{2}$ control charts were based on the $\mathrm{UV}-\mathrm{Vis}$ analysis. The wavelength range (404-416 nm) was chosen based on the characteristics of the absorption band of nanoparticles associated with the presence of surface plasmons and for silver nanoparticles in the range of 400-440 nm [18, 19].

\section{Results and Discussion}

\section{Effect of the Tannic Acid Concentration and Temperature}

The effects of the molar ratio of tannic acid to silver ions and effect of temperature on the degree of silver ions conversion, as well as the size and dispersion of the resulting nanoparticles, were examined Fig. 3 presents 

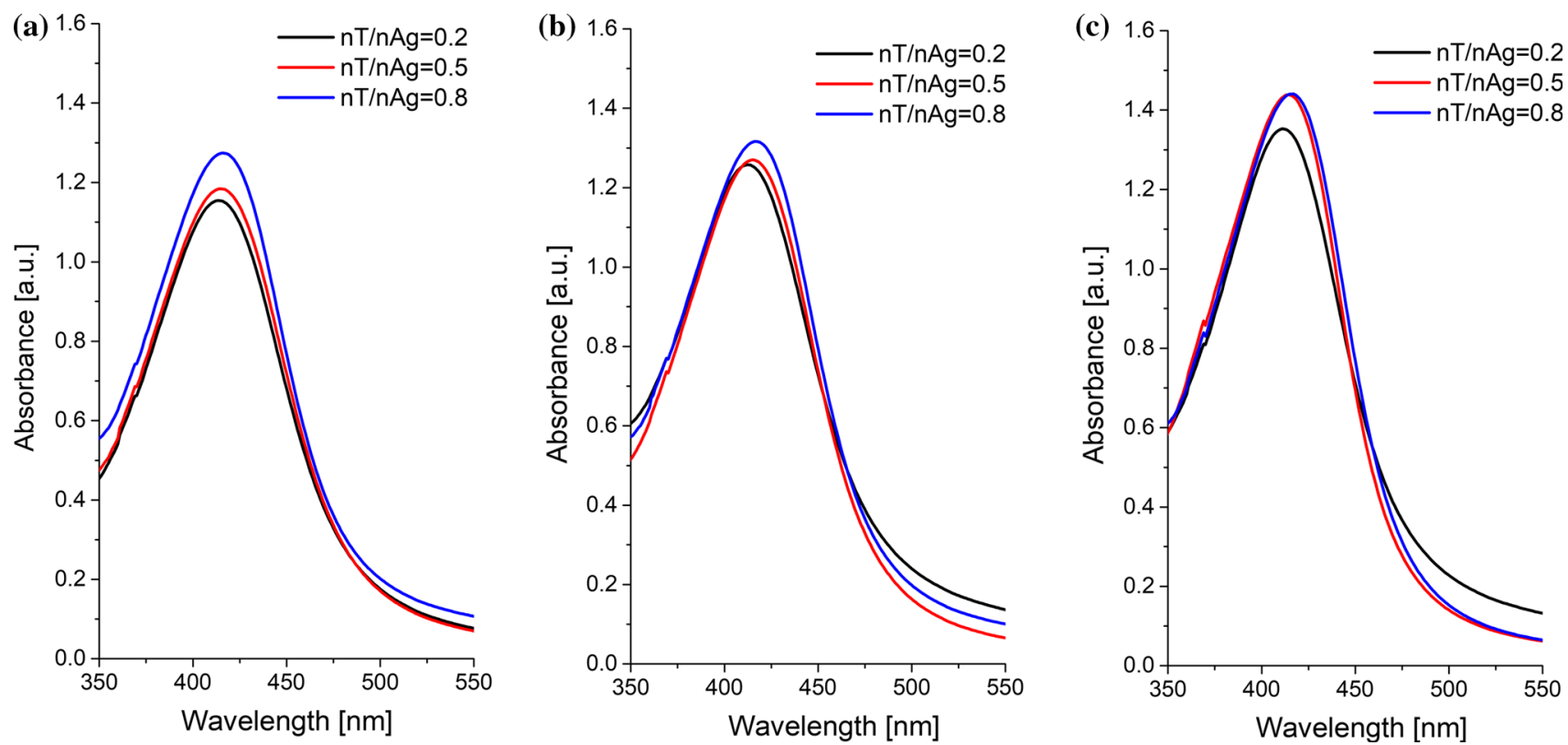

Fig. 3 UV-Vis plot nanosilver (the samples were collected at the 45th min of the process) for different molar ratio tannic acid to silver ions and in temperature: a $20^{\circ} \mathrm{C}, \mathbf{b} 45^{\circ} \mathrm{C}, \mathbf{c} 70^{\circ} \mathrm{C}$

spectrum plot of silver nanoparticles for different process conditions. The symmetry of the peaks, especially no blur of the peaks towards the larger wavelengths, indicated a homogeneous distribution of the size of the nanoparticles. The AgNPs plasmonic response, presented on the figure, are depends on size and shape of particles, dielectric environment, and on mutual electromagnetic interactions among particles in close adjacency [20]. Correlating the AgNPs plasmonic properties with their morphology, by UV-visible spectroscopy is a fast and easy way for in situ monitoring of the nanosilver synthesis. This is very useful, for instance, in the control processes, while the stability and quality of the particles is specially required. Other methods of nanoparticles characterisation as transmission electron microscopy analysis, even though present more information on products, require many additional preparation stages, which could modify samples. For $\mathrm{nT} / \mathrm{nAg}$ equals 0.5 , the size distribution analysis (Fig. 4) revealed high homogeneity of samples over the whole processing time. The largest particle size distribution was noticed in processes running at $70{ }^{\circ} \mathrm{C}$. Higher divergences in diameters may result from uneven energy transport resulting from laminar flow in the reactor.

The obtained values of average particle diameter, conversion of silver ions and zeta electrokinetic potential are presented in Table 2. The measurement of the zeta electrokinetic potential was performed for exemplary samples. The total stability of the system is influenced by two types of stabilisation of silver nanoparticles: steric stabilisation and electrochemical stabilisation. As a result of the presence of large molecules, tannic acid prevented the silver particles from being in close contact, causing the formation of the so-called steric effect [21]. On the other hand, the system was stabilised as a result of electrostatic stabilisation, which is described by the zeta potential. The presence of sodium hydroxide affected the $\mathrm{pH}$ of the system, which
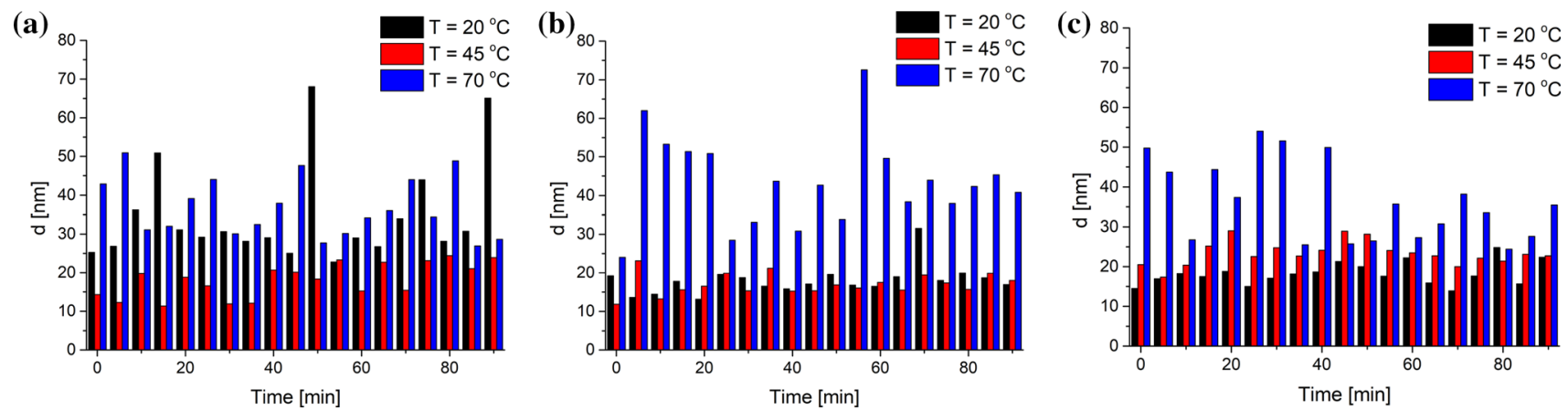

Fig. 4 Plot of nanoparticles size distribution in different temperatures for molar ratio tannic acid to silver ions: a $0.2: 1$, b $0.5: 1$, c $0.8: 1$ 
Table 2 Average size of nanosilver, conversion of silver ions and electrokinetic potential analysis depending on temperature and molar ratio of tannic acid to silver ions

\begin{tabular}{|c|c|c|c|c|c|c|c|}
\hline \multirow[t]{2}{*}{$\mathrm{nT} / \mathrm{nAg}$} & \multicolumn{3}{|c|}{ Average particle diameter $[\mathrm{nm}]$} & \multicolumn{3}{|c|}{ Conversion of silver ions $\alpha_{\mathrm{Ag}^{+}}[\%]$} & \multirow[t]{2}{*}{$\zeta[\mathrm{mV}]$} \\
\hline & $20\left[{ }^{\circ} \mathrm{C}\right]$ & $45\left[{ }^{\circ} \mathrm{C}\right]$ & $70\left[{ }^{\circ} \mathrm{C}\right]$ & $20\left[{ }^{\circ} \mathrm{C}\right]$ & $45\left[{ }^{\circ} \mathrm{C}\right]$ & $70\left[{ }^{\circ} \mathrm{C}\right]$ & \\
\hline $0.2: 1$ & $34.8 \pm 12.7$ & $18.2 \pm 4.3$ & $29.5 \pm 6.2$ & $99.88 \pm 0.09$ & $99.96 \pm 0.05$ & $99.93 \pm 0.52$ & -34.1 \\
\hline $0.5: 1$ & $18.3 \pm 3.8$ & $17.1 \pm 2.7$ & $43.4 \pm 11.4$ & $99.75 \pm 0.02$ & $99.79 \pm 0.13$ & $99.96 \pm 0.33$ & -20.7 \\
\hline $0.8: 1$ & $18.2 \pm 2.8$ & $23.3 \pm 6.9$ & $39.5 \pm 19.3$ & $99.08 \pm 0.02$ & $99.16 \pm 0.03$ & $99.85 \pm 0.09$ & -11.1 \\
\hline
\end{tabular}

affects the value of the zeta potential. Combining both phenomena, it was noticed that the higher concentration of tannic acid, the lower $\mathrm{pH}$ of the system and, consequently, the lower zeta potential. At a constant concentration of sodium hydroxide, as the concentration of tannic acid in the solution increases, the proportion of reactions between tannic acid and hydroxide increases, which limits the effect of $\mathrm{NaOH}$ on silver nanoparticles. However, despite the lower proportion of electrostatic stabilisation, the increase in the concentration of tannic acid contributes to the increase in the steric effect, hence the stability of the system is preserved.

To check the stability of the colloids, after 3 months the solutions of silver nanoparticles were again analysed with UV-Vis spectroscopy (Fig. 5). Silver nanoparticles produced in $20{ }^{\circ} \mathrm{C}$ with different tannic molar ratio to silver ions are presented. Overtime nanoparticles where $\mathrm{nT} / \mathrm{nAg}$ was equal to 0.5 or 0.8 were still stable. Only small changes towards slight increased particle sizes were observed. Overtime nanosilver with tannic to silver ions molar ratio equal 0.2 was characterised with lower spectrum what confirms lower stability of product compared to other samples.

A comparison of the influence of temperature and molar ratio of tannic acid to silver ions was performed using a two-factor analysis of variance. Table 3 summarises the results of the analysis. The parameters significantly influencing particle diameter and the degree of conversion were the temperature and temperature interaction with the change in the molar ratio of tannic acid to silver ions (nT/ $\mathrm{nAg}$ ) what confirmed $p$ value (probability value) below 0.05 . The increase in temperature caused both an increase in the conversion rate and an increase in average particle diameter. The molar ratio of $\mathrm{nT} / \mathrm{nAg}$ significantly affected the degree of conversion, causing a decline. In the case of particle diameter analysis, the effect of changes in the molar ratio $\mathrm{nT} / \mathrm{nAg}$ was not confirmed, which may result from a sufficient concentration of tannic acid even when the molar ratio $\mathrm{nT} / \mathrm{nAg}$ was lowest. The effect of temperature and the effect of the molar ratio of tannic acid to silver ions on mean diameter of particles and conversion rate of silver ions are shown in Fig. 6. Comparing the
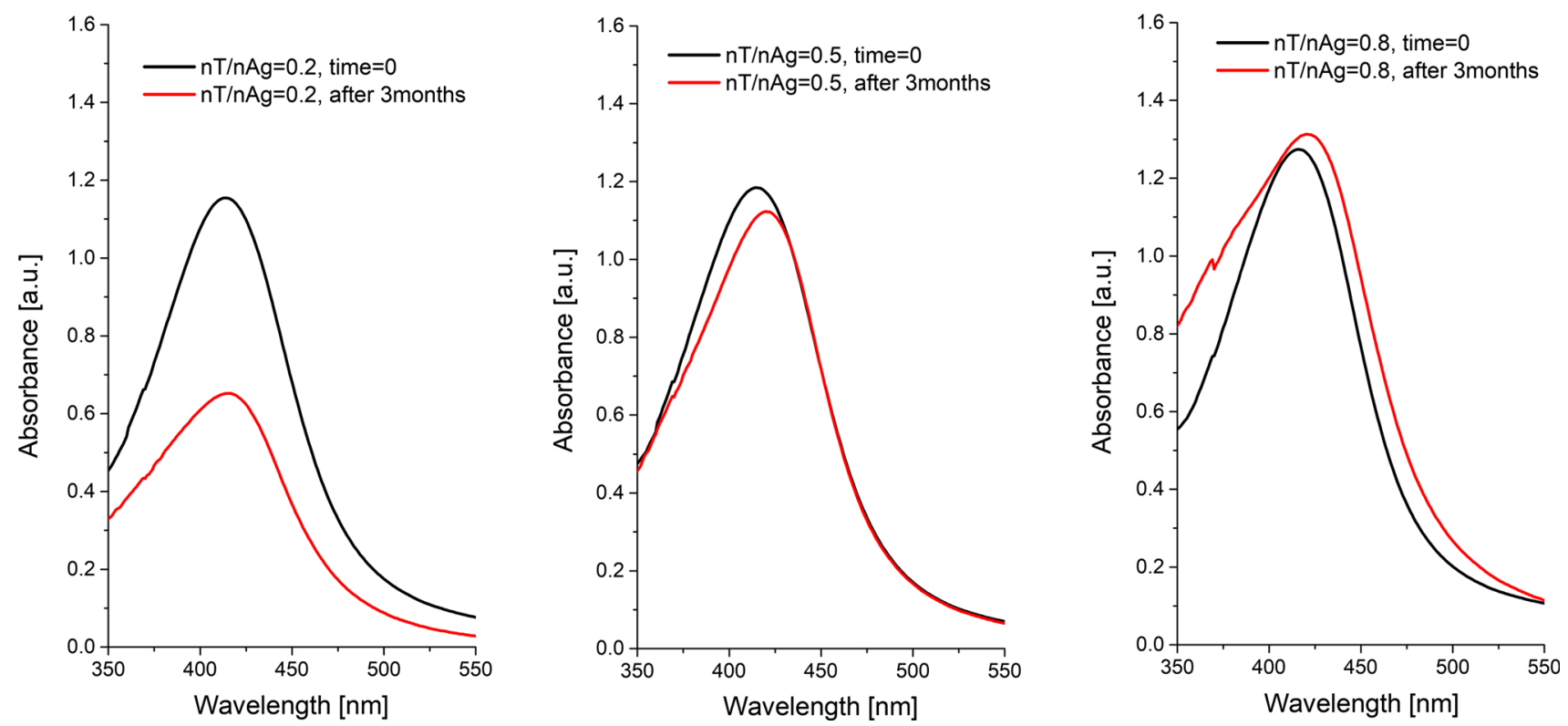

Fig. $5 \mathrm{UV}-$ Vis plot nanosilver in temperature $20^{\circ} \mathrm{C}$ for different molar ratio tannic acid to silver ions just after process and after 3 months 
Table 3 Univariate tests of significance for average size of nanosilver and for conversion of silver ions
SS (sum of squares) df (degree of freedom) $\quad$ MS (mean square) $\quad$ F-value $\quad p$ value

Average particle diameter [nm]

\begin{tabular}{llllll} 
Intercept & 109,528 & 1 & $1.1 \cdot 10^{5}$ & 2026 & 0.0000 \\
$\mathrm{nT} / \mathrm{nAg}$ & 216.8 & 2 & 108.4 & 2.005 & 0.1380 \\
$\mathrm{~T}\left[{ }^{\circ} \mathrm{C}\right]$ & 5078 & 2 & 2539 & 46.97 & 0.0000 \\
$\mathrm{nT} / \mathrm{nAg} * \mathrm{~T}$ & 10,067 & 4 & 2516 & 46.56 & 0.0000 \\
Error & 8757 & 162 & 54.1 & & \\
Conversion & of & silver ions $(-)$ & & & \\
Intercept & 170.1 & 1 & 170.1 & $3.5 \cdot 10^{8}$ & 0.0000 \\
$\mathrm{nT} / \mathrm{nAg}$ & 0.0011 & 2 & 0.0005 & 111 & 0.0000 \\
$\mathrm{~T}\left[{ }^{\circ} \mathrm{C}\right]$ & 0.0003 & 2 & 0.0001 & 26 & 0.0000 \\
$\mathrm{nT} / \mathrm{nAg} * \mathrm{~T}$ & 0.0004 & 4 & 0.0001 & 20 & 0.0000 \\
Error & 0.0008 & 162 & 0.0000 & & \\
\hline
\end{tabular}
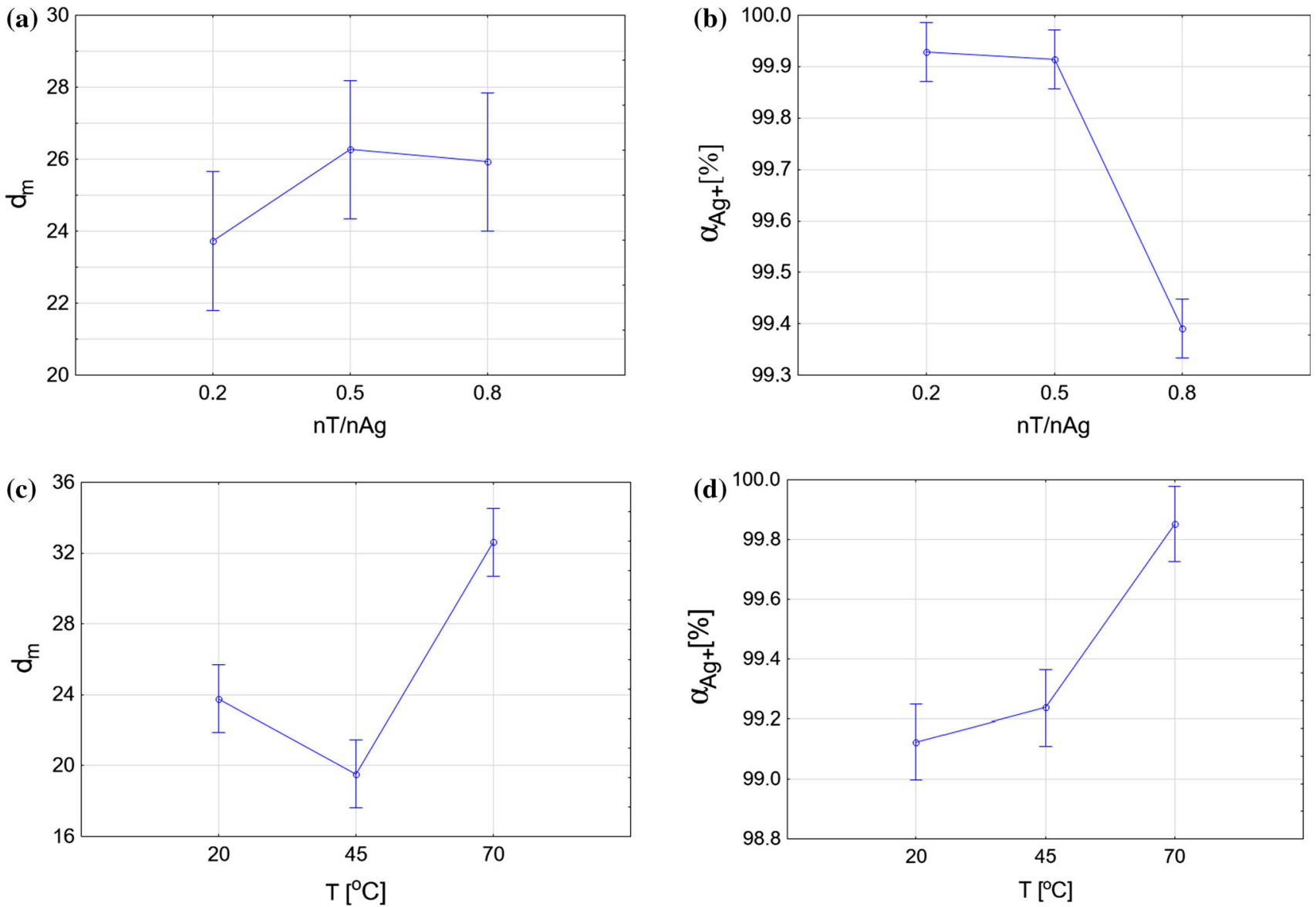

Fig. 6 The diagram of the effect: a of the molar ratio tannic acid to silver ions for mean diameter of particles, $\mathbf{b}$ of the molar ratio tannic acid to silver ions for conversion rate of $\mathrm{Ag}^{+} \mathbf{c}$ of the temperature for mean diameter of particles, $\mathbf{d}$ of the temperature for conversion rate of $\mathrm{Ag}^{+}$

results with the Uv-Vis analysis, slight changes in the obtained spectra are visible. With the increase of the molar ratio of $n T / n A g$, the shift of the nanosilver peak towards longer wavelengths is noticeable. This confirms the increase in particle size. Additionally, it can be seen that, as the temperature rises, the absorbance at constant $\mathrm{nT} / \mathrm{nAg}$ increases (Fig. 6), what suggest that the temperature affects the degree of process conversion. 


\section{Multidimensional Control Charts}

The main challenge associated with the continuous process is to maintain stable reactor operation. It is convenient to use control charts for process control and observation of deviations in process variables [22]. Control charts, also known as Shewhart charts, are an Statistical Process Control (SPC) tool that allows effective supervision of the process, observation of changing trends in it, and to take appropriate corrective actions in a sufficient time frame as to not allow them to exceed the acceptable limits of the controlled parameters [23, 24].

The stability of the continuous process was examined on the basis of UV-Vis and DLS analysis. The UV-Vis method is a method that directly allows to study the quality of the process of silver nanoparticles creation and, together with the control charts, detect incompatibilities caused by poor reactor operation. In the UV-Vis spectrum, a characteristic absorption peak for silver nanoparticles is visible in the $400-440 \mathrm{~nm}$ range. The maximum absorbance depends on the shape, particle size, aggregation level, etc. [17]. For the nanosilver-tannic acid system, control charts were prepared for each process using data collected from the UV-Vis analysis in the wavelength range 404-416 nm. Chart analysis suggested that a stable process was obtained in processes in which the ratio of tannic acid to silver ions was 0.5 and 0.8 over the entire temperature range. At the smallest concentration of the reducer, the process shows instability, which was also confirmed in the DLS studies.

Examples of control charts for the process of creating silver nanoparticles, depending on the molar ratio of tannic acid to silver ions and temperature, are presented in Fig. 7. Hotelling 2T control charts evaluate stability of processes using multiple related quality characteristics and show differing samples from each series. X-bar charts and $\mathrm{R}$ charts present the samples means and the samples ranges of particles size with upper and lower control lines. As long as the plotted points are between the extreme lines, the system remains stable. Processes in which $\mathrm{nT} / \mathrm{nAg}$ was 0.5 , despite different temperatures, are characterized by good stability. The smallest dispersion of points was obtained for the process in which the molar ratio of tannic acid to silver ions was $0.5: 1$, the process was carried out at $20{ }^{\circ} \mathrm{C}$. Therefore, in order to obtain a stable working system of the reactor and to obtain silver nanoparticles with the same parameters, the process should be carried out for the given conditions. Additionally, control charts of $\mathrm{X}$ and $\mathrm{R}$ show that the differences between measurements are the lowest for the $20{ }^{\circ} \mathrm{C}$ which suggests choosing this system as the most stable one.

The process carried out in a continuous system requires the selection of appropriate parameters ensuring good product quality as well as checking how the impact of parameters affects the stability of the reactor operation and the reproducibility of the results. Simultaneous analysis of the influence of parameters and the course of the control charts suggests that the parameters in which the reactor works stably, the conversion rate reaches sufficient values, and the size distribution of the nanoparticles is as low as possible are $20{ }^{\circ} \mathrm{C}$ or $45^{\circ} \mathrm{C}$ and $\mathrm{nT} / \mathrm{nAg}$ equal 0.5 . For the presented data, the control charts are characterised by the smallest spread, and there are no points outside the range of system stability. The $\mathrm{nT} / \mathrm{nAg}$ ratio of $0.2 \mathrm{did}$ not result in stable reactor operation. In turn, $\mathrm{nT} / \mathrm{nAg}$ at 0.8 reduced the conversion rate. In addition, a lower concentration of tannic acid is preferred due to the reduction of manufacturing costs of nanosilver.

In this research, the data collected by UV-Vis and DLS were used to determine the stability of the process. Control charts based on UV-Vis are characterised by direct results and the method is cheaper compared to the DLS method. On this basis, it can be suggested that the UV-Vis method can be successfully used to test the control of the continuous formation of silver nanoparticles.

Figures 8, 9 and 10 show the UV-Vis spectra, size distribution by intensity and TEM images of nanosilver colloid prepared in tubular reaction under the optimum reaction parameters: $\mathrm{nT} / \mathrm{nAg}=0.5$ and $\mathrm{T}=20^{\circ} \mathrm{C}$. Figure 9 confirmed uniform size of nanoparticles. The particles have spherical shape in the range of about 5-40 nm (depending on the process parameters). The particle size histogram was obtained by measuring at least 100 NPs. Based on histogram, silver nanoparticles were characterised by high homogeneity and spherical shape. The range of obtained diameters was between 3 and $10 \mathrm{~nm}$, for all particles (under the optimum reaction parameters). Analysis of EDX spectra of sample with $\mathrm{nT} / \mathrm{nAg}$ equalled 0.5 synthesized in $20^{\circ} \mathrm{C}$. The nanosilver particles (bright contrast) are verified also by EDX. In the spectra of area Fig. 9, which includes bright spots, there is a clear peak of Ag confirming its presence. Additionally, presence of sodium was detected. The $\mathrm{Cu}$ peaks, presented on spectra, come from the carbon coated copper foil that was used to achieve the electron microscopy images. Size of nanoparticles achieved by DLS method showed bigger particles (range of 14-60 nm). The dynamic light scattering method presents the hydrodynamic diameters of the particles including outer layer of the solvent particles. Hence the diameter results obtained from the TEM analysis are smaller compared to the diameter obtained by the DLS method. The UV-Vis absorption spectra of these nanosilver colloids revealed a high intense plasmon absorption peak near $415 \mathrm{~nm}$, what is characteristic for silver nanoparticles $[18,19]$. The changes of position and shape of the absorption spectrum of the nanoparticles were not 


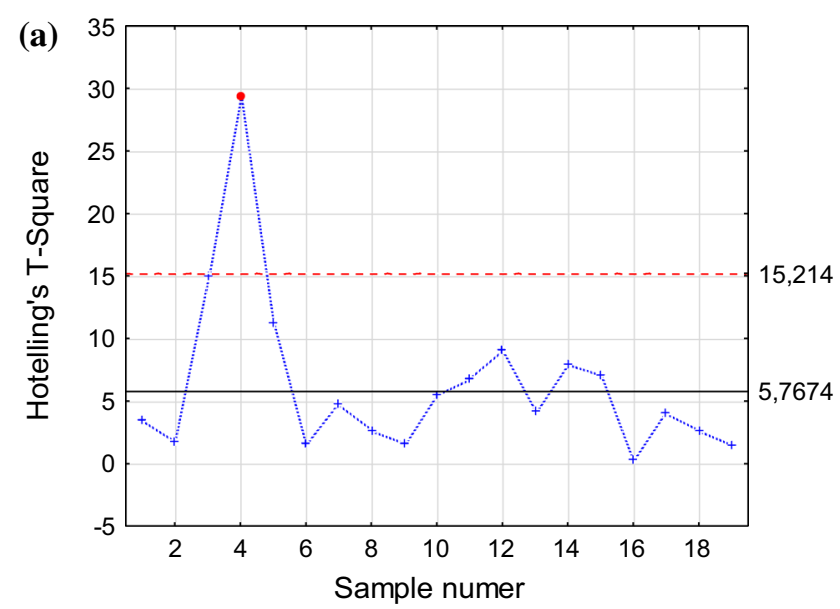

$X$ and Moving R Chart; variable: $n T / n A g=0.2, T=20 \cdot C$ Histogram of

Observations
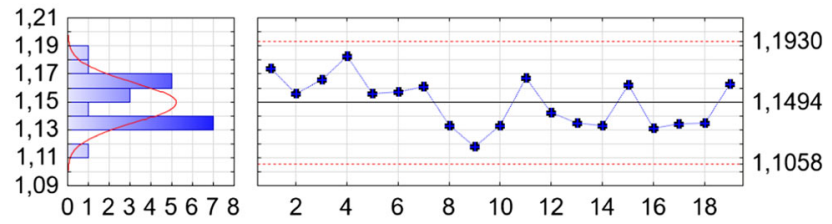

Histogram of Moving Ranges
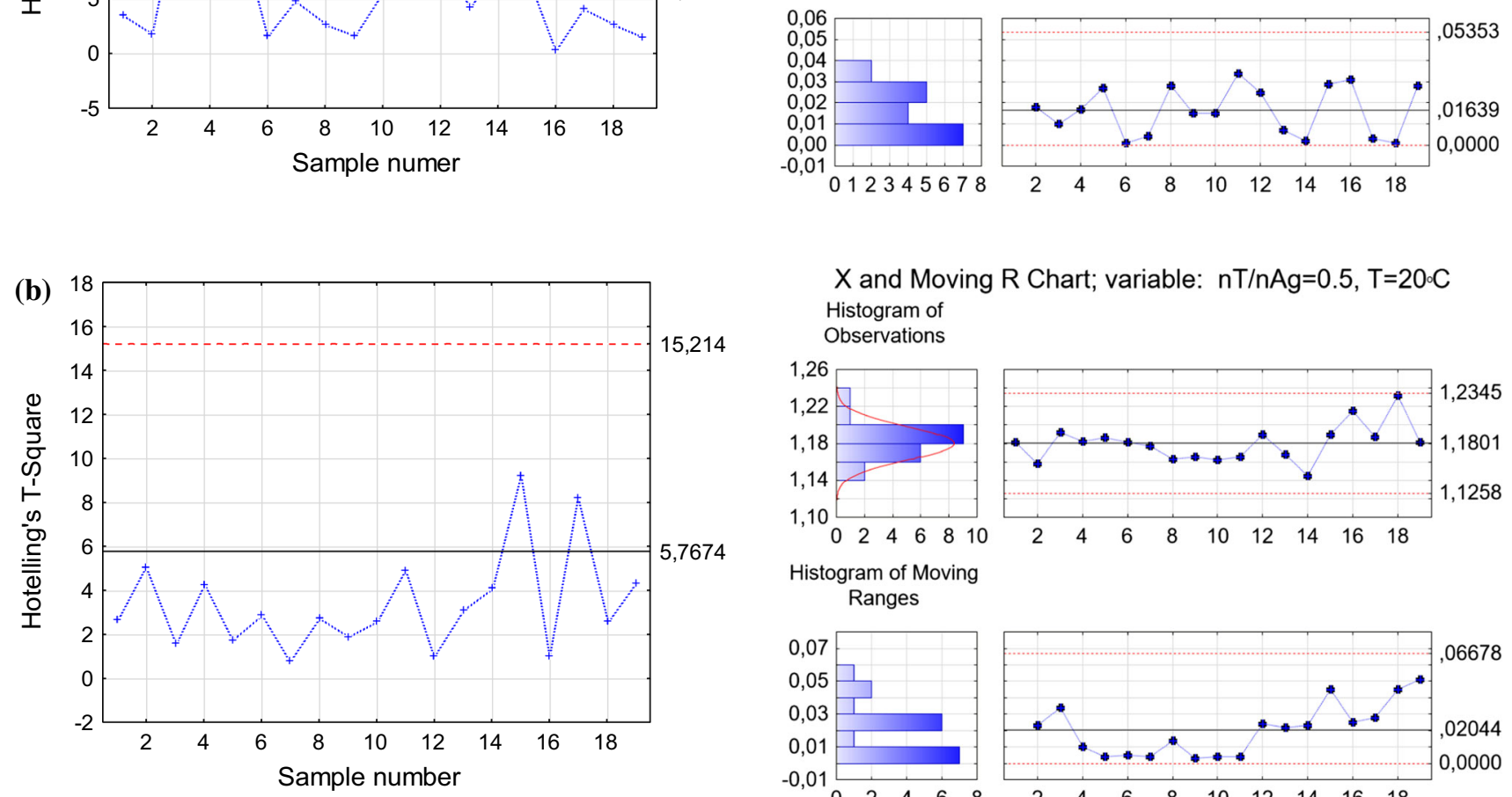

$X$ and Moving R Chart; variable: $\mathrm{nT} / \mathrm{nAg}=0.5, \mathrm{~T}=20^{\circ} \mathrm{C}$ Histogram of

Observations
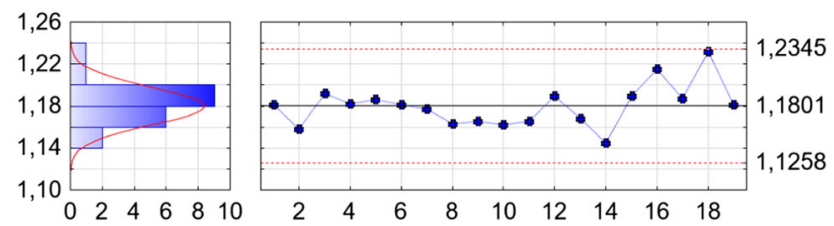

Histogram of Moving

Ranges
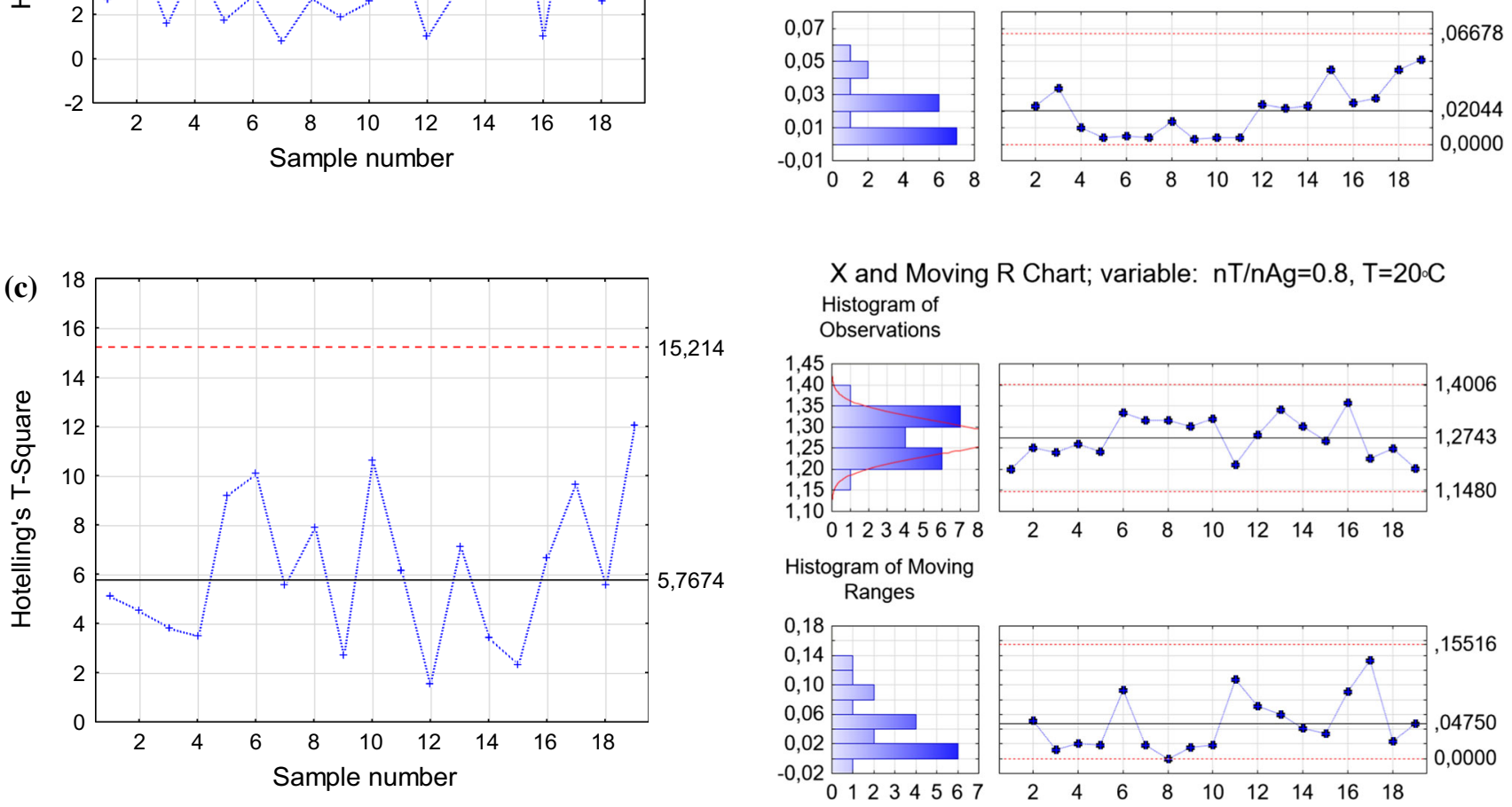

Fig. 7 The control charts for silver nanoparticles synthesis process in $\mathrm{T}=20^{\circ} \mathrm{C}: \mathbf{a} \mathrm{nT} / \mathrm{nAg}=0.2: 1.0, \mathbf{b} \mathrm{nT} / \mathrm{nAg}=0.5: 1.0, \mathbf{c} \mathrm{nT} / \mathrm{nAg}=0.8: 1.0$ 
Fig. $8 \mathrm{UV}-\mathrm{Vis}$ plot nanosilver (the samples were collected every $10 \mathrm{~min}$ through $30 \mathrm{~s}$ during $90 \mathrm{~min}$ reactor operation) for molar ratio tannic acid to silver ions $0.5: 1$ in $20^{\circ} \mathrm{C}$
Fig. 9 Size distribution by intensity for molar ratio tannic acid 0.5:1 and temperature $20{ }^{\circ} \mathrm{C}$
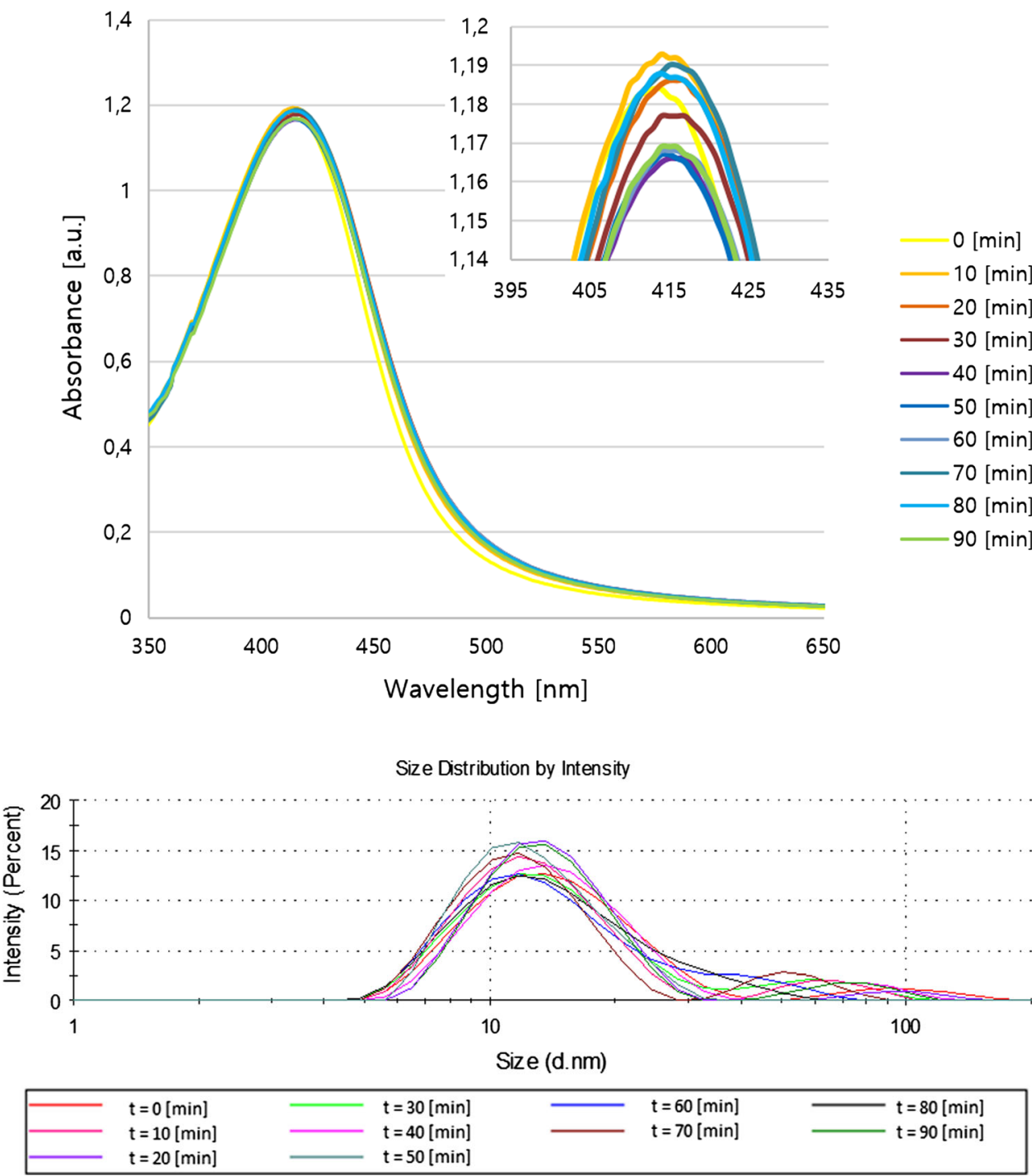

observed. Stable absorption spectrum over time confirmed homogenous particles distribution and constant concentration of silver nanoparticles.

\section{Principles of Green Chemistry}

According to the principles of green chemistry, substances should be safe for the environment in addition to their primary functions $[25,26]$. In the process of nanoparticles production, scientists search compounds which, apart from non-toxic properties, would both act as reducer and stabiliser, [19, 27]. Such compounds include organic chemical compounds from the group of unsaturated polyhydric alcohols, such as ascorbic acid, or compounds from the group of phenolic acids, like tannic acid or gallic acid [28].

Anastas and Warner defined green chemistry as the utilization of a set of principles that reduces or eliminates the use or generation of hazardous substances in the design, manufacture and application of chemical products [29]. In order to define the scope and nature of green chemistry, they distinguished 12 principles of green chemistry.

1. Prevention It is better to prevent the formation of pollution and waste than to dispose of it. The continuous process limits the production of waste associated even with the washing of equipment, which cannot be avoided in periodic processes. In addition, the control of appropriate parameters and constant process control allows immediate reaction in case of accidents and, thereby, prevents the formation of undesired products.

2. Atom Economy It is desirable to design such synthesis methods in order to allow maximum use and incorporation into the final product of all materials used in the process. The calculations confirmed by the tests showed that the degree of substrate conversion was over $99 \%$. This shows that the feed material is fully reacted. The product obtained was characterised by high purity (no silver ions). 
Fig. 10 Size distribution histograms of AgNPs, TEM images with EDX collective spectrum and mapping of obtained silver nanoparticles in $20{ }^{\circ} \mathrm{C}$ for molar ratio tannic acid to silver ions $0.5: 1$
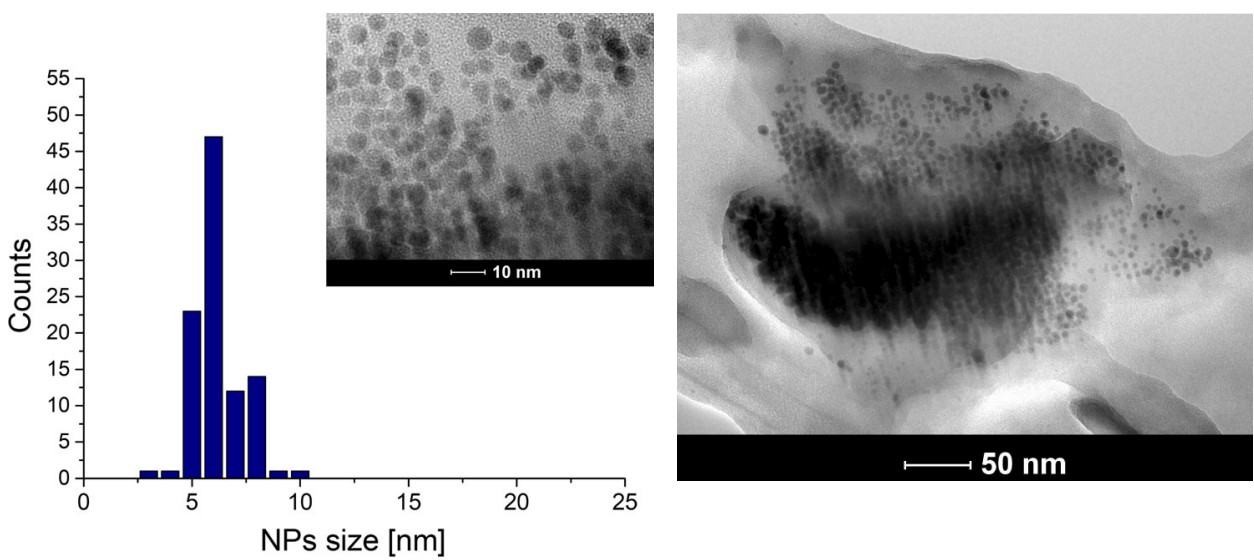

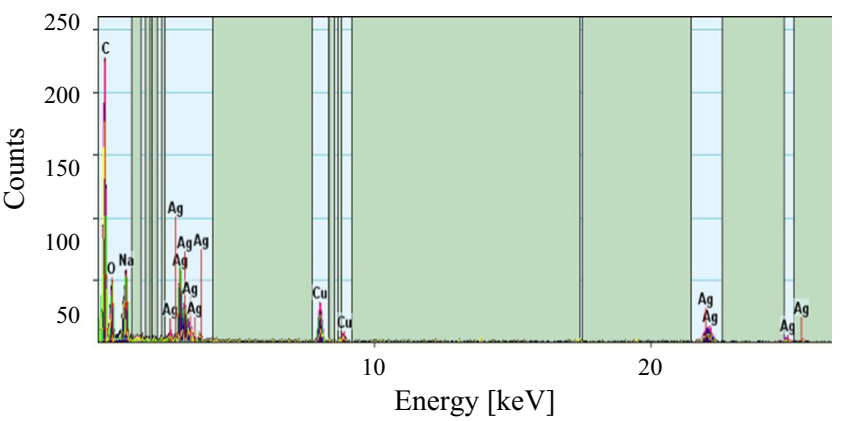

exceed $45{ }^{\circ} \mathrm{C}$, similar results were previously obtained at ambient temperature, which is an advantage of the method.

7. Use of Renewable Feedstocks In the process, tannic acid acted as a reducer and stabiliser. As a renewable raw material, tannic acid is usually extracted from plants, e.g. Rhus coriaria and Rhus chinensis [34].

8. Reduce Derivatives It is necessary to limit processes in which derivatives of a product may be generated, thereby increasing the amount of by-products and waste products. This process eliminated the use of additional reagents. Stabilising properties were obtained using tannic acid, which prevents agglomeration of nanosilver.

9. Catalysis The use of catalysts aims to reduce energy consumption, improve selectivity, or enhance process efficiency. In this work, hydroxyl ions were added to the system. The addition of $\mathrm{OH}^{-}$ions have a catalytic effect, significantly speeding up the reaction.

10. Design for Degradation Chemical materials after their period of use should not pose a threat to the environment. Tannic acid has natural origins and is biodegradable. Silver nanoparticles that are not stabilised by tannic acid will agglomerate, making their oxidation impossible.

11. Real-Time Analysis for Pollution By running the process in a continuous system, it is possible to control the process "in situ". In-line analysis using control charts allows continuous production monitoring and the ability to 
react immediately to fluctuations in the process. Thus, it is possible to maintain a constant level of product quality, without changing the size or shape of the particles. Control charts continuously evaluate the process.

12. Inherently Safer Chemistry for Accident Prevention If possible, reagents and production methods should be chosen in order to minimise the risk of accidents. In this process, flammable and toxic reactants such as hydrazine or sodium borohydride were eliminated, replaced by biodegradable tannic acid. The apparatus protects against the release of substances to the environment, limiting the contact of workers with reagents to a minimum. The importance of process control was confirmed, among others, in the works Polte [35] and Thünemann [36].

In the presented work, the process of continuous nanosilver production using tannic acid as a reducer and stabiliser meets all 12 principles of green chemistry. SPC techniques allow for continuous process monitoring and control of product properties. A rapid response in case of lack of stability in the process is necessary to obtain a reproducible product.

\section{Conclusions}

The presented work analysed the continuous process of silver nanoparticles production in a tubular reactor. Twofactor analysis of the variance showed the effect of tannic acid concentration and temperature on the transformation of silver ions and the size of silver nanoparticles. Temperatures of $20^{\circ} \mathrm{C}$ and $45{ }^{\circ} \mathrm{C}$ and a 0.5 molar ratio of tannic acid to silver provided the best parameter values. Increases in temperature had a positive effect on the degree of conversion of the resulting silver nanoparticles. In addition, this increase resulted in obtaining nanoparticles with larger diameters. To analyse the stability of the process, control charts based on the UV-Vis and DLS methods were used. The analysis of control charts showed that the continuous process of creating nanosilver with the optimised choice of parameters was a stable process. Process control using the UV-Vis test and Hotelling T2 control charts permitted control over the process. In the event of instability of reactor operation, this enables immediate observation of any process changes, which contributes to improving and maintaining the quality of the final product. The presented method can be more easily combined with other installations (for example adding silver nanoparticles, to give other products biocidal properties).

From the presented analysis it can be concluded that the described method of nanosilver synthesis meets the principles of green chemistry. The reducing-stabilising properties of tannic acid and its natural origin means that this silver nanoparticles preparation method is environmentally friendly. The use of a dual-acting reagent first, does not require the addition of compounds that could influence on the final product and allows to obtain suspension with high concentration nanoparticles, e.g. 500 ppm. Furthermore, constant process conditions reduce energy consumption which limits process costs. UV-Vis techniques with the control charts allow for in-line analysis. Continuous process monitoring enables for better control of product parameters and rapid response in the event of deviations from the stable work of the system.

Funding This research did not receive any specific grant from funding agencies in the public, commercial, or not-for-profit sector.

\section{Compliance with Ethical Standards}

Conflict of interest The authors report no declarations of interest.

Open Access This article is distributed under the terms of the Creative Commons Attribution 4.0 International License (http://creative commons.org/licenses/by/4.0/), which permits unrestricted use, distribution, and reproduction in any medium, provided you give appropriate credit to the original author(s) and the source, provide a link to the Creative Commons license, and indicate if changes were made.

\section{References}

1. S. Prabhu and E. K. Poulose (2012). Int. Nano Lett. 2, (32-3), 3. https://doi.org/10.1186/2228-5326-2-32.

2. X. F. Zhang, Z. G. Liu, W. Shen, and S. Gurunathan (2016). Int. J. Mol. Sci. 17, 1534-1568. https://doi.org/10.3390/ ijms 17091534.

3. K. K. Kuorwel, M. J. Cran, J. D. Orbell, S. Buddhadasa, and S. W. Bigger (2015). Compr. Rev. Food Sci. Food Saf. 14, 411-430. https://doi.org/10.1111/1541-4337.12139.

4. A. Hebeish, A. El-Shafei, S. Sharaf, and S. Zaghloul (2014). Carbohydr. Polym. 103, 442-447. https://doi.org/10.1016/j.carb pol.2013.12.050.

5. J. Fu, L. Mo, Y. Li, W. Li, W. Li, J. Ran, X. Fan, X. Zhao, and L. $\mathrm{Li}$, The 8th Annual IEEE International Conference on Nano/ Micro Engineered and Molecular Systems (IEEE; 2013), pp. 619-622.

6. H. L. Yang, J. C. Lin, and C. Huang (2009). Water Res. 43, 3777-3786. https://doi.org/10.1016/j.watres.2009.06.002.

7. X. Tang, D. Guo, S. Jiang, N. Gu, Y. Ni, J. Ge, and Q. Hu (2017). J. Nanosci. Nanotechnol. 17, 1046-1052. https://doi.org/10.1166/ jnn.2017.12671.

8. K. Ranoszek-Soliwoda, E. Tomaszewska, E. Socha, P. Krzyczmonik, A. Ignaczak, P. Orlowski, M. Krzyzowska, G. Celichowski, and J. Grobelny (2017). J. Nanopart. Res. 19, 273. https://doi.org/10.1007/s11051-017-3973-9.

9. A. Tufail (2014). J. Nanotechnol. 2014, (1), 1-11. https://doi.org/ $10.1155 / 2014 / 954206$.

10. K. S. Chou, Y. C. Chang, and L. H. Chiu (2012). Ind. Eng. Chem. Res. 51, 4905-4910.

11. R. Baber, L. Mazzei, N. T. K. Thanh, and A. Gavriilidis (2015). RSC Adv. 116, 1-7. https://doi.org/10.1039/C5RA17466J. 
12. C. Y. Tai, Y. H. Wang, C. T. Tai, and H. S. Liu (2009). Ind. Eng. Chem. Res. 48, 10104-10109. https://doi.org/10.1021/ie9005645.

13. K. J. Hartlieb, M. Saunders, R. J. J. Jachuck, and C. L. Raston (2010). Green Chem. 12, 1012-1017. https://doi.org/10.1039/ C000708K.

14. J. Huang, L. Lin, Q. Li, D. Sun, Y. Wang, Y. Lu, N. He, K. Yang, X. Yang, H. Wang, W. Wang, and W. Lin (2008). Ind. Eng. Chem. Res. 47, 6081-6090. https://doi.org/10.1021/ie701698e.

15. S. T. He, Y. L. Liu, and H. Maeda (2008). J. Nanoparticle Res. 10, 209-215. https://doi.org/10.1007/s11051-008-9509-6.

16. J. B. Deshpande and A. A. Kulkarni (2015). Chem. Eng. Technol. 41, 157-167.

17. D. A. Cristaldi, F. Yanar, A. Mosayyebi, P. Garćı-Manrique, E. Stulz, D. Carugo, and X. Zhang (2018). New Biotechnol. 25, 1-7. https://doi.org/10.1016/j.nbt.2018.02.002.

18. G. L. Flower, S. V. Latha, and K. V. Rao (2016). J. Mol. Liq. 221, 333-338. https://doi.org/10.1016/j.molliq.2016.06.017.

19. P. Bollella, C. Schulz, G. Favero, F. Mazzei, R. Ludwig, and L. Gorton (2017). R. Electroanal. 29, 77-86. https://doi.org/10. 1002/elan.201600476.

20. V. Amendola, O. M. Bakr, and F. Stellacci (2010). Plasmonics 5, (1), 85-97. https://doi.org/10.1007/s11468-009-9120-4.

21. R. X. He, R. Liang, P. Peng, and Y. N. Zhou (2017). J. Nanoparticle Res. 19, 267-277. https://doi.org/10.1007/s11051017-3953-0.

22. L. Liu and L. Liu (2018). IEEE Trans. Ind. Electron. 65, 5893. https://doi.org/10.1109/TIE.2017.2777416.

23. N. A. Saleh, M. A. Mahmoud, M. J. Keefe, and W. H. Woodall (2015). J. Qual. Technol. 47, 127-138. https://doi.org/10.1080/ 00224065.2015.11918120.

24. S. Sukparungsee, S. Kuvattana, P. Busababodhin, and Y. Areepong (2018). Commun. Stat. Simul. Comput. 47, 413-419. https://doi.org/10.1080/03610918.2016.1228958.

25. M. H. El-Rafie, H. B. Ahmed, and M. K. Zahran (2014). Carbohydr. Polym. 107, 174-181. https://doi.org/10.1016/j.carbpol. 2014.02.024.
26. B. Wei, Z. Shi, J. Xiao, Y. Xu, and L. Lv (2017). J. Biomater. Tissue Eng. 7, 418-425. https://doi.org/10.1166/jbt.2017.1581.

27. R. Shanmuganathan, Ali D. Mubarak, D. Prabakar, H. Muthukumar, N. Thajuddin, S. S. Kumar, and A. Pugazhendhi (2017). Environ. Sci. Pollut. Res. 25, 1-9. https://doi.org/10.1007/ s11356-017-9367-9.

28. S. Francis, S. Joseph, E. P. Koshy, and B. Mathew (2017). Environ. Sci. Pollut. Res. 24, 17347-17357. https://doi.org/10.1007/ s11356-017-9329-2.

29. P. T. Anastas and J. C. Warner Green Chemistry: Theory and Practice (Oxford University Press, Oxford, 1998).

30. M. K. Zahran, H. B. Ahmed, and M. H. El-Rafie (2014). Carbohydr. Polym. 111, 10-17. https://doi.org/10.1016/j.carbpol. 2014.03.029.

31. H. E. Emam and H. B. Ahmed (2016). Carbohydr. Polym. 135, 300-307. https://doi.org/10.1016/j.carbpol.2015.08.095.

32. L. Ge, Q. Li, M. Wang, J. Ouyang, X. Li, and M. M. Q. Xing (2014). Int. J. Nanomed. 9, 2399-2407. https://doi.org/10.2147/ IJN.S55015.

33. C. Levard, E. M. Hotze, G. V. Lowry, and G. E. Brown (2012). Environ. Sci. Technol. 46, 6900-6914. https://doi.org/10.1021/ es2037405.

34. A. Jayanegara, G. Goel, H. P. S. Makkar, and K. Becker (2015). Anim. Feed Sci. Technol. 209, 60-68. https://doi.org/10.1016/j. anifeedsci.2015.08.002.

35. J. Polte, R. Erler, A. F. Thünemann, S. Sokolov, T. T. Ahner, K. Rademann, F. Emmerling, and R. Kraehnert (2010). Resolution. ACS Nano 4, 1076-1082. https://doi.org/10.1021/nn901499c.

36. A. F. Thünemann, S. Rolf, P. Knappe, and S. Weidner (2009). Anal. Chem. 81, 296-301. https://doi.org/10.1021/ac802009q.

Publisher's Note Springer Nature remains neutral with regard to jurisdictional claims in published maps and institutional affiliations. 